\title{
Molecular, Dynamic, and Structural Origin of Inhomogeneous Magnetization Transfer in Lipid Membranes
}

\author{
Scott D. Swanson, ${ }^{1 *}$ Dariya I. Malyarenko, ${ }^{1}$ Mario L. Fabiilli, ${ }^{1}$ Robert C. Welsh, ${ }^{1}$ \\ Jon-Fredrik Nielsen, ${ }^{2}$ and Ashok Srinivasan ${ }^{1}$
}

Purpose: To elucidate the dynamic, structural, and molecular properties that create inhomogeneous magnetization transfer (ihMT) contrast.

Methods: Amphiphilic lipids, lamellar phospholipids with cholesterol, and bovine spinal cord (BSC) specimens were examined along with nonlipid systems. Magnetization transfer (MT), enhanced MT (eMT, obtained with double-sided radiofrequency saturation), ihMT (MT - eMT), and dipolar relaxation, $\mathrm{T}_{1 \mathrm{D}}$, were measured at 2.0 and $11.7 \mathrm{~T}$.

Results: The amplitude of inMT ratio (ihMTR) is positively correlated with $T_{1 D}$ values. Both inMTR and $T_{1 D}$ increase with increasing temperature in BSC white matter and in phospholipids and decrease with temperature in other lipids. Changes in inMTR with temperature arise primarily from alterations in MT rather than eMT. Spectral width of MT, eMT, and inMT increases with increasing carbon chain length.

Conclusions: Concerted motions of phospholipids in white matter decrease proton spin diffusion leading to increased proton $\mathrm{T}_{1 \mathrm{D}}$ times and increased inMT amplitudes, consistent with decoupling of Zeeman and dipolar spin reservoirs. Molecular specificity and dynamic sensitivity of inMT contrast make it a suitable candidate for probing myelin membrane disorders. Magn Reson Med 77:1318-1328, 2017. (c) 2016 International Society for Magnetic Resonance in Medicine

Key words: magnetization transfer; myelin membrane; white matter; lipid dynamics

\section{INTRODUCTION}

Magnetization transfer (MT) between water and immobilized molecules in vivo alters image contrast in MRI and provides a method to indirectly image the semisolid components in tissue (1-4). MT partially saturates the longitudinal—or Zeeman-magnetization of the semisolid spin system and transfers this depletion to water protons by

\footnotetext{
${ }^{1}$ Department of Radiology, University of Michigan, Ann Arbor, Michigan, USA.

${ }^{2}$ Department of Biomedical Engineering, University of Michigan, Ann Arbor, Michigan, USA.

Grant sponsor: United States National Center for Advancing Translational Sciences, National Institutes of Health; Grant number: UL1TR000433; Grant sponsor: Department of Radiology, University of Michigan.

*Correspondence to: Scott D. Swanson, Ph.D., Department of Radiology, The University of Michigan, 3208A Medical Science I, Ann Arbor, MI 481095030. E-mail: sswanson@umich.edu

Received 14 August 2015; revised 6 February 2016; accepted 18 February 2016

DOI $10.1002 / \mathrm{mrm} .26210$

Published online 30 March 2016 in Wiley Online Library (wileyonlinelibrary. com).
}

(C) 2016 International Society for Magnetic Resonance in Medicine

chemical, proton, or magnetic exchange (5). For clinical research, MR images are typically acquired with and without radiofrequency (RF) saturation and a computed MT ratio (MTR) image is generated. More comprehensive studies provide quantitative estimates of the concentration of semisolid components in tissue (4), which has been histologically correlated to myelin content $(6,7)$.

For many tissues, the semisolid resonance is homogeneously broadened by fast and efficient intra- and intermolecular spin diffusion mediated by strong magnetic dipolar coupling $(8,9)$. Hence, RF saturation either with positive or with negative off-resonance affects all semisolid protons equally. Model MT systems such as gelatin, cross-linked bovine serum albumin, and agarose show this behavior, where a more rigid structure and variable proton-proton distances create a homogeneously broadened semisolid spectrum with Gaussian shape. Motions and structure of neuronal tissue, specifically myelin, are different. Fast rotation of carbon chains about the lipid director axis reduces intramolecular dipolar interactions, and molecular chain diffusion removes intermolecular dipolar terms (10-12). The proton-proton distances in lipids are more uniform than those in proteins and are dominated by the $1.78 \AA$ spacing of methylene $\mathrm{CH}_{2}$ protons, creating a maximum rigid-lattice dipolar coupling of $21.28 \mathrm{kHz}$ (splitting of $31.9 \mathrm{kHz}$ ). This coupling is reduced by molecular motion within the lipid membrane, characterized by an order parameter between 0 and 1 (13-15).

Furthermore, protein content in neuronal tissues varies widely: $30 \%$ in myelin and $55 \%$ in gray matter (16). Due to the dynamic and structural aspects of neuronal lipids, inhomogeneous broadening is expected to be more pronounced in protein-depleted white matter (WM) than in protein-rich gray matter (GM).

The combination of phospholipids, sterols, and proteins in neuronal tissues $(16,17)$ create the well-known lipid bilayer system. Synthetic phospholipid membranes systems are easily fabricated and widely used as surrogate models of in vivo membranes. NMR studies of model phospholipid systems (18-21) implicate cholesterol and galacto-cerebrosides as specific molecular conduits for cross-relaxation and generation of MT. Solid-state NMR studies of biological membranes $(22,23)$ reveal distinct anisotropy (orientation dependence) of the proton-proton dipolar interaction and corresponding inhomogeneous broadening of the proton semisolid resonance (24).

Recent studies have shown that applying double-sided RF saturation at both positive and negative off-resonance 
frequencies generates an enhanced magnetization transfer (eMT) between water and inhomogeneously broadened semisolid components. The signal intensity differences between MT (single-sided RF saturation) and eMT (double-sided RF saturation) are collectively termed inhomogeneous magnetization transfer (ihMT $=$ MT eMT) and are most prominently observed in myelinated tissue such as WM $(25,26)$. The enhanced RF saturation has been attributed to the presence of magnetization with dipolar order, which can be decoupled from the Zeeman magnetization with double-sided RF saturation (27-29). To establish a quantitative relation between measured ihMT parameters and tissue properties, optimize acquisition protocol for clinical studies, and ultimately infer specific disease mechanisms, the molecular origins of ihMT need to be characterized.

This work explores the relationship between ihMT and the molecular structure and dynamics of lipid membranes. Following Lee et al. (29), a brief treatment of the coupling and decoupling between dipolar and Zeeman spin system is presented for chemical systems with long and short proton $\mathrm{T}_{1 \mathrm{D}}$ times. We report here the dependency between temperature, ihMT signal amplitude, and the decay time of dipolar order, $\mathrm{T}_{1 \mathrm{D}}$, in tissue specimens, and in model molecular systems. We examined the magnitude and spectral width of ihMT in several lipid samples of different carbon chain lengths and different molecular inclusions (e.g. cholesterol). We also measured MT, eMT, and $\mathrm{T}_{1 \mathrm{D}}$ in immobilized proteins and polysaccharides. These model systems provide molecular analogues of myelin membrane components. Changing temperature affects molecular motion in lipids and allows investigation of related changes in the values of MT, eMT, ihMT, and $\mathrm{T}_{1 \mathrm{D}}$. These experiments elucidate how the molecular dynamics and structure of lipids are tied to generation of ihMT in vivo. In addition, we studied the effects of carbon chain length and the presence of cholesterol on MT and ihMT in relation to membrane order parameter $(12,14,15,24)$.

\section{THEORY}

Provotorov Theory Under Single-Sided and Double-Sided RF Saturation

Provotorov theory of RF saturation in strongly dipolar coupled spin systems helps explain the dependence of ihMT on proton $\mathrm{T}_{1 \mathrm{D}}$ (29-31). Outlined by Goldman (32), developed for MT by Yeung et al. (27) and Morrison et al. (33), and applied by Lee et al. (29) to understand enhanced RF saturation in liquid crystals and by Varma et al. (34) to explain ihMT, Provotorov theory shows that a system of immobilized, dipolar coupled spins possesses magnetization with both Zeeman and dipolar order. In a static magnetic field, the Zeeman and dipolar terms of the Hamiltonian commute and no energy is exchanged. Single-sided RF saturation couples the Zeeman and dipolar terms, allowing an exchange of energy between the reservoirs (35). Whereas the RF acts to saturate the populations of spin energy levels of the semisolid component, both $\mathrm{T}_{1}$ and $\mathrm{T}_{1 \mathrm{D}}$ processes act to restore magnetization to the steady state. Double-sided $\mathrm{RF}$ saturation, at both positive and negative off-reso- nance frequencies, removes the coupling between Zeeman and dipolar magnetizations $(25,29,32)$. Under dual frequency $R F$ saturation, $\mathrm{T}_{1 \mathrm{D}}$ is eliminated and only $\mathrm{T}_{1}$ processes restore magnetization to steady state. With the $\mathrm{T}_{1 \mathrm{D}}$ removed, $\mathrm{RF}$ saturation is more efficient, resulting in eMT of the semisolid component. Therefore, the ihMT experiment is explained as a difference between coupled Zeeman and dipolar reservoirs with single-sided RF saturation (MT) and decoupled reservoirs with double-sided RF saturation (eMT).

Coupling between Zeeman and dipolar order can be more easily appreciated and visualized if Equation 8 in Lee et al. (29) is solved, setting $W=W^{\prime}=R_{R F}^{b}$ and $\Delta$ $=\Delta^{\prime}$ for single-sided RF saturation. Under these conditions, the Zeeman and dipolar reservoirs are coupled and Zeeman longitudinal magnetization of the semisolid component at steady state is

$$
M_{b}^{s a t}\left(\Delta, \omega_{1}\right)=\frac{\omega_{l o C}^{2}+2 \Delta^{2} R_{R F}^{b} T_{1 D}}{\omega_{l o c}^{2}+2 \omega_{l o c}^{2} R_{R F}^{b} T_{1}+2 \Delta^{2} R_{R F}^{b} T_{1 D}},
$$

where $\omega_{l o c}$ is the local dipolar magnetic field, $\Delta$ is the off-resonance frequency of the RF pulse, $T_{1}$ is $T_{1}$ of the semisolid component, $R_{R F}^{b}=\frac{1}{2} \omega_{1}^{2} g_{b}(\Delta)$ is the rate of saturation of the semisolid protons, and $g_{b}(\Delta)$ is the normalized shape of the semisolid proton resonance, here assumed to be Gaussian (1).

Similarly, setting $\Delta=-\Delta^{\prime}$ for double-sided RF saturation (and assuming that RF saturation rates are equal at $\Delta$ and $-\Delta^{\prime}$, valid for the symmetric dipolar Hamiltonian) decouples the dipolar reservoir from the Zeeman reservoir. The Zeeman magnetization of the semisolid component is then

$$
M_{b}^{s a t}\left(\Delta, \omega_{1}\right)=\frac{1}{1+2 R_{R F}^{b} T_{1}} .
$$

All influence of the dipolar reservoir is removed from the saturation profile.

Figure 1a shows the results of calculating the b-spin magnetization with $\omega_{\text {loc }}=\sqrt{1 / 3\left(T_{2}^{b}\right)^{2}}, T_{2}^{b}=12 \mu s, \omega_{1}=$ $2 \pi 300 \frac{\mathrm{rad}}{\mathrm{s}}, T_{1}=0.1 \mathrm{~s}$, and $T_{1 D}=20 \mathrm{~ms}$. The local field, $\omega_{l o c}$, is set to the theoretical value for a Gaussian line (32). The difference between Equations [1] and [2] produces an ihMT spectrum as a function of $\Delta$. When $\mathrm{T}_{1 \mathrm{D}}$ is short $(200 \mu \mathrm{s}$ in Fig. $1 \mathrm{~b})$, terms in Equation 1 with $\mathrm{T}_{1 \mathrm{D}}$ approach zero, and both single-sided and doublesided RF saturation are described by Equation 2. The physical consequence of this is that when $\mathrm{T}_{1 \mathrm{D}}$ is short, dipolar order is quickly dissipated, the resonance line is homogeneously broadened, and ihMT will be very small or zero (Fig. 1b). Conversely, long proton $\mathrm{T}_{1 \mathrm{D}}$ times will lead to large values of ihMT (Fig. 1a).

A fit of Provotorov theory to experimental data is shown in Figure 1C. A sample of $10 \%(\mathrm{w} / \mathrm{w})$ Prolipid 161 (described hereafter) was studied with MT and eMT pulse sequences with a steady state RF field of $\omega_{1}=2 \pi$ $369 \frac{\mathrm{rad}}{\mathrm{s}}$ from -50 to $50 \mathrm{kHz}$ in $1-\mathrm{kHz}$ increments. An ihMT profile was calculated as the difference between the two saturation profiles and fitted to the difference of Equations [1] and [2], yielding the fitted parameters 

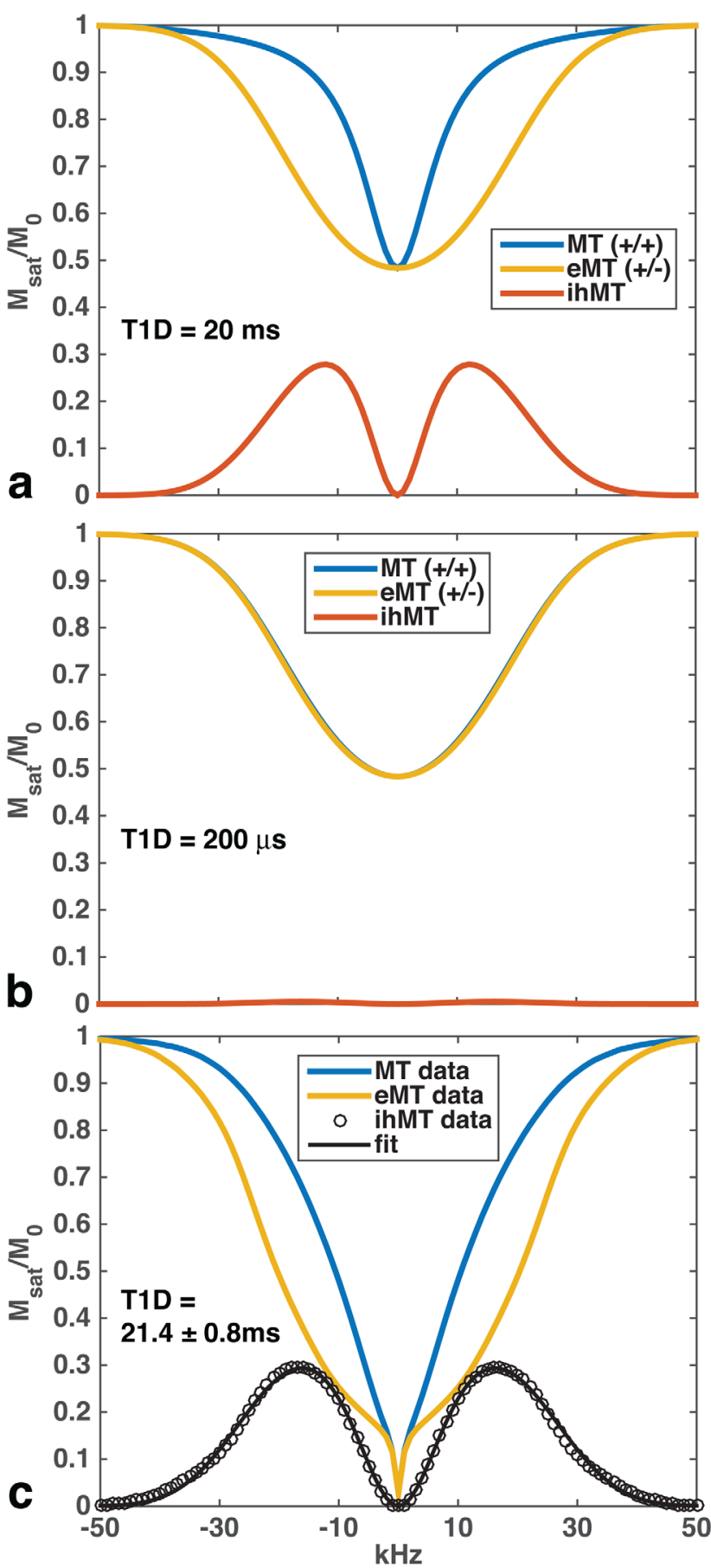

FIG. 1. (a, b) Simulation of MT (blue), eMT (yellow), and inMT (rust) spectra for $T_{1 D}=20 \mathrm{~ms}$ (a) and $T_{1 D}=200 \mu \mathrm{s}$ (b) according to Provotorov formalism shown in Equations [1] and [2]. ihMT is large when $T_{1 D}$ is long and minimal when $T_{1 D}$ is short. (c) An example of fitting Provotorov theory to inMT data collected from a sample of Prolipid 161. These simulations and fittings show that Provotorov theory helps explain the additional MT generated by dualfrequency RF saturation.

$T_{2}^{b}=10.92 \pm 0.12 \mu \mathrm{s}, T_{1}=30.6 \pm 1.4 \mathrm{~ms}$, and $T_{1 D}=21.2 \pm$ $0.6 \mathrm{~ms}$. $\omega_{l o c}$ is set to the Gaussian value as before. Figure 1C shows that Provotorov theory of RF saturation describes the salient features of ihMT for model lipid systems such as Prolipid 161.

\section{METHODS}

Sample Preparation

A bovine spinal cord (BSC) was obtained from a local slaughterhouse, fixed in $10 \%$ neutral-buffered formalin (Sigma-Aldrich, St. Louis, Missouri, USA), and embedded in $1.5 \%$ agarose. To measure tissue $\mathrm{T}_{1 \mathrm{D}}, 5-\mathrm{mm}$ cubes of white and GM were dissected from the specimen and soaked in $\mathrm{D}_{2} \mathrm{O}$ to replace exchangeable water protons. Similarly, hyaline cartilage was dissected from the femoral condyle of chicken legs obtained at a local grocery store and bathed in $\mathrm{D}_{2} \mathrm{O}$ to replace exchangeable water protons.

Type A Gelatin from porcine skin (G2500, 300 bloom; Sigma-Aldrich) was added to $\mathrm{H}_{2} \mathrm{O}$ at $15 \%$ w/w. Following hydration, the slurry was slowly heated in a microwave oven until melted. The hot solution was poured into 15-mL centrifuge tubes and allowed to cool and gel. Aqueous samples of $1.5 \%(\mathrm{w} / \mathrm{w})$ agarose were made in a similar fashion.

Prolipid 161 (PL161; Ashland Specialty Ingredients, Wilmington, Delaware, USA), composed of cetearyl alcohol, behenyl alcohol, and hydroxyethyl cetearamidopropyldimonium chloride, forms a lamellar lipid bilayer when mixed with water. The wax-like PL161 and $600 \mathrm{mM} \mathrm{NiCl}_{2}$ (to reduce $\mathrm{T}_{1}$ ) were heated to $85^{\circ} \mathrm{C}$ and mixed at $15 \%(\mathrm{w} / \mathrm{w})$ PL161. An additional sample was made in $\mathrm{D}_{2} \mathrm{O}$.

Multilamellar phospholipid vesicles (MLVs) were prepared by dissolving dipalmitoyl phosphatidylcholine (DPPC) (Avanti Polar Lipids, Alabaster, Alabama, USA) and cholesterol (Sigma-Aldrich) in chloroform in a vial. A thin lipid film, obtained via evaporation of chloroform in a vacuum oven, was rehydrated with $\mathrm{D}_{2} \mathrm{O}$. The mixture was then vortexed to create and suspend MLVs in $\mathrm{D}_{2} \mathrm{O}$. The liposomes were subjected to four freeze/thaw cycles to assure minimum vesicle size. The final composition was $500 \mathrm{mg} \mathrm{D}_{2} \mathrm{O}, 55 \mathrm{mg}$ DPPC, and $35 \mathrm{mg}$ cholesterol. Approximately $300 \mu \mathrm{L}$ of product was transferred to a glass test tube for NMR study at 11.7 T. The mean particle size diameter of the DPPC:cholesterol (DPPC:Ch) sample, determined by Coulter counter (Multisizer 4, Beckman Coulter, Brea, California, USA), was $2.15 \pm 1.75 \mu \mathrm{m}$. These large MLV structures hinder rotational diffusion (36) and result in negligible narrowing of the NMR resonance from vesicle rotation.

A lamellar liquid crystal (LLC) sample was made with (weight percent) sodium dodecyl sulfate (23\%), 1-decanol $(12 \%)$, and $\mathrm{D}_{2} \mathrm{O}(65 \%)$ as described previously (30). In short, a proper amount of sodium dodecyl sulfate was dissolved in $\mathrm{D}_{2} \mathrm{O}$ and decanol was swiftly mixed into the solution to create the LLC. The sample was vortexed, heated, subjected to several freeze thaw cycles, centrifuged, and aged 1 week to assure sample homogeneity.

\section{Data Acquisition}

\section{MRI In Vitro}

BSC images were acquired on a 2.0 T MRI system (Varian Unity/Inova) equipped with Acustar S-180 gradients. A spin-echo MRI pulse sequence was modified to include an MT RF pulse train with two independently controlled frequencies (Fig. 2a). Four sets of images were acquired 


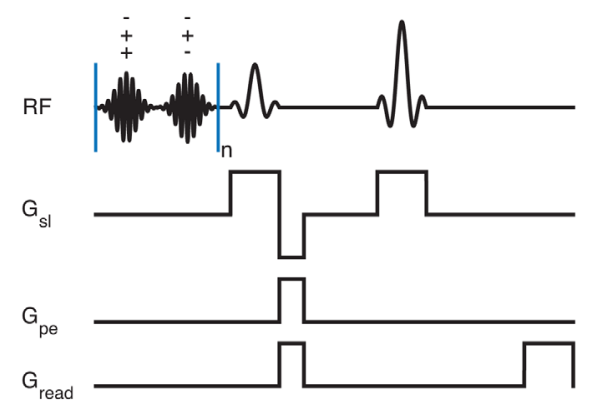

a
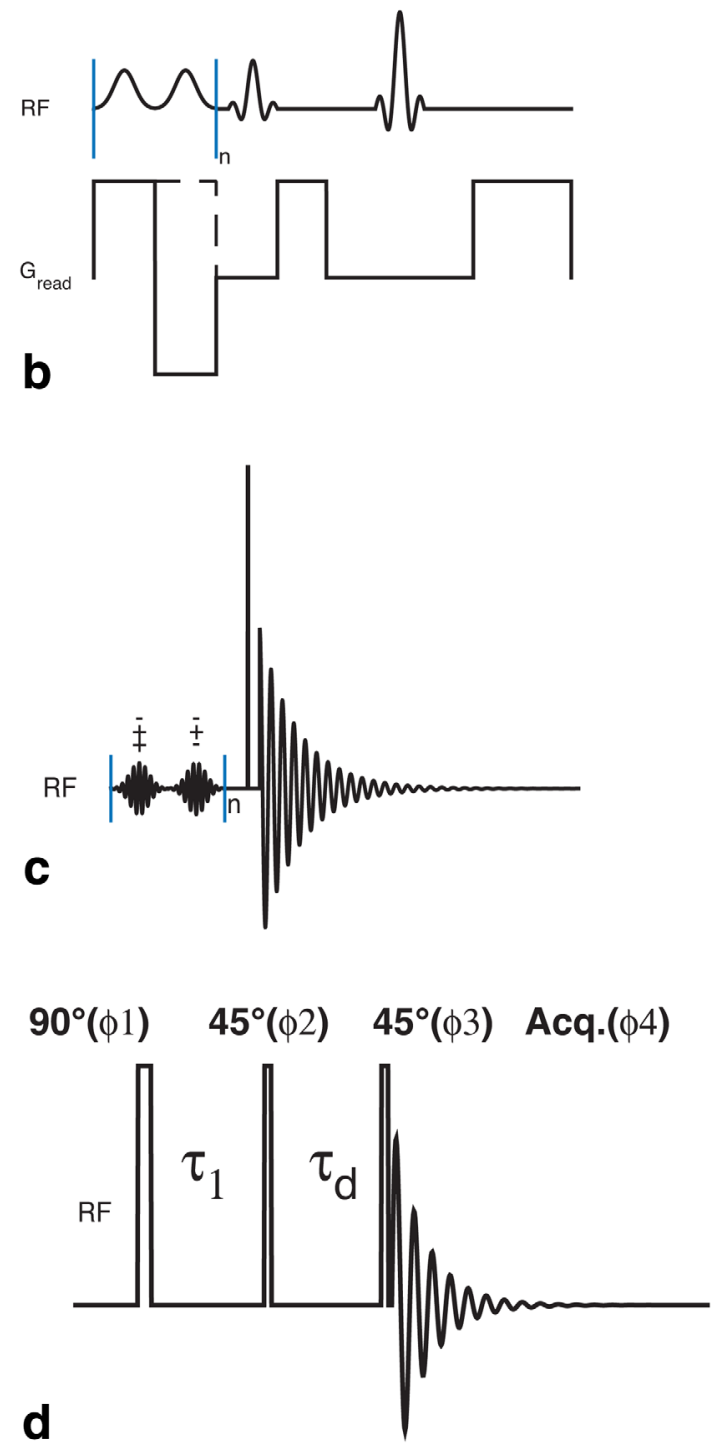

FIG. 2. MT and eMT pulse sequences for MRI and NMR studies. (a) BSC imaging. (b) Single-shot MT and eMT for protonated samples. (c) Solid-state proton NMR with MT and eMT for deuterated samples. (d) Proton $T_{1 D}$ measurement in deuterated samples. To eliminate multiple quantum and Zeeman order coherences in proton $\mathrm{T}_{1 \mathrm{D}}$ measurements, eight-step quadrature phase cycling was set to (d) $\phi 1=00112233$, $\phi 2=11221122, \phi 3=02020202$, and $\phi 4=20200202$.

with no $\mathrm{RF},(+/+) \mathrm{RF}$ saturation, (-/-) RF saturation, and $(+/-) \mathrm{RF}$ saturation. The duration of the 5-sigma Gaussian-shaped RF pulses was $500 \mu$ s, and the pattern was repeated 2000 times for each line of k-space. The peak $\mathrm{B}_{1}$ amplitude was $20.4 \mu \mathrm{T}$ and root mean square (RMS) $B_{1}$ was $10.8 \mu \mathrm{T}$. TR was $2.1 \mathrm{~s}$ and TE $11 \mathrm{~ms}$. Slice thickness was $5 \mathrm{~mm}$ and in-plane resolution was $0.2 \mathrm{~mm}$. Four averages were acquired, and imaging time for the entire set was $84 \mathrm{~min}$. Images were acquired at $20^{\circ} \mathrm{C}$ and $40^{\circ} \mathrm{C}$ by changing the temperature of gradient coil cooling water. ROI contours were drawn (MATLAB R2014b; MathWorks, Natick, Massachusetts, USA) for GM and $\mathrm{WM}$, and average and standard deviations were computed (Table 1).

\section{Single-Shot MT, eMT, and ihMT of Protonated Model Systems}

Data for samples made in $\mathrm{H}_{2} \mathrm{O}$ in $15 \mathrm{~mL}$ centrifuge tubes (gelatin, agarose, and PL161) were acquired at $2.0 \mathrm{~T}$ with the aid of a home-built, Alderman-Grant MRI probe (37). MT for all off-resonance frequencies was generated by a single-shot MT (ssMT) spectroscopy sequence (38). eMT was generated by a variant of the ssMT sequence where the z-gradient direction is reversed to alternatively expose spins to positive and negative RF offsets (Fig. 2b). A pair of 5-ms Gaussian-shaped RF pulses (peak amplitude $18.8 \mu \mathrm{T}$, RMS amplitude $10.2 \mu \mathrm{T}$ ) was repeated 200 times for an RF duration of 2 s. ssMT data were normalized to a one-dimenstional image with no $\mathrm{RF}$ saturation. ihMT was measured as the difference between regular MT, (+/+) Gz, and eMT, (+/-) Gz. Sample temperature was controlled by regulated heated air flow. MT, eMT, and ihMT in PL161 were measured as a function of temperature from $5^{\circ} \mathrm{C}$ to $85^{\circ} \mathrm{C}$. MT and eMT were computed as $100 \times\left(M_{\text {sat }}^{+/+} / M_{0}\right)$ and $100 \times\left(M_{\text {sat }}^{+/-} / M_{0}\right)$, respectively, and ihMT $=(\mathrm{MT}-\mathrm{eMT})$. Note that MT differs from MTR (=100 - MT, etc.).

\section{Solid-State NMR, MT Spectroscopy of Samples in $\mathrm{D}_{2} \mathrm{O}$}

Data for samples made in $\mathrm{D}_{2} \mathrm{O}$ were acquired in 12-mm test tubes with the aid of a home-built, four-turn solenoid NMR probe tuned to $85.54 \mathrm{MHz}$. Solid-state proton NMR spectra of the model lipid samples were acquired using a 4- $\mu$ s RF pulse length, $200 \mathrm{kHz}$ spectral width. RF saturation in PL161 and LLC samples was acquired with 25 off-resonance frequencies and sampled logarithmically from $100 \mathrm{kHz}$ to $100 \mathrm{~Hz}$ (Fig. 2c). A matrix of saturation frequencies and powers was created to enable $(+/$ $+),(-/-)$, and (+/-) RF saturation. Data were obtained at $20^{\circ} \mathrm{C}$. The offset frequency dependence for difference spectra of LLC and PL161 samples was measured as a function $B_{1}$ field strength. The maximum ihMT occurred at $31.0 \mu \mathrm{T}$ for PL161 and at $1.32 \mu \mathrm{T}$ for LLC. DPPC:Ch data were collected on a Varian 11.7 T high-resolution NMR spectrometer (Varian Inc., Palo Alto, California, USA).

Jeener-Broekaert Proton $T_{1 D}$ Measurements of Samples in $\mathrm{D}_{2} \mathrm{O}$

A Jeener-Broekaert sequence, $90^{\circ}(\phi 1)-\tau_{1}-45^{\circ}(\phi 2)-$ $\tau_{d}-45^{\circ}(\phi 3)-A c q(\phi 4)$, was used to measure the decay of dipolar order $\left(\mathrm{T}_{1 \mathrm{D}}\right.$ ) (Fig. $2 \mathrm{~d}$ ). $\mathrm{RF}$ and receiver phase cycling over eight acquisitions was used to eliminate 
Table 1

Percent MTR, eMTR, and ihMTR Measured at $\pm 10 \mathrm{kHz}$ RF Saturation

\begin{tabular}{|c|c|c|c|c|c|}
\hline Material & Temperature $\left( \pm 1^{\circ} \mathrm{C}\right)$ & MTR & eMTR & ihMTR & $\mathrm{T}_{1 \mathrm{D}}, \mathrm{ms}^{\mathrm{a}}$ \\
\hline \multicolumn{6}{|l|}{$\mathrm{BSC}^{\mathrm{b}}$} \\
\hline GM & $20^{\circ} \mathrm{C}$ & $63.7 \pm 0.8$ & $65.9 \pm 0.8$ & $2.1 \pm 0.4$ & $1.59 \pm 0.57$ \\
\hline WM & $20^{\circ} \mathrm{C}$ & $65.3 \pm 2.2$ & $68.2 \pm 2.1$ & $2.9 \pm 0.5$ & $1.90 \pm 0.20$ \\
\hline GM & $40^{\circ} \mathrm{C}$ & $59.7 \pm 0.9$ & $64.4 \pm 0.7$ & $4.7 \pm 0.9$ & $4.06 \pm 1.20$ \\
\hline WM & $40^{\circ} \mathrm{C}$ & $52.4 \pm 2.6$ & $62.9 \pm 2.5$ & $10.5 \pm 0.7$ & $11.1 \pm 1.8$ \\
\hline \multicolumn{6}{|l|}{ Samples $( \pm 0.15)^{\mathrm{c}}$} \\
\hline Gelatin (15\%) & $22^{\circ} \mathrm{C}$ & 24.0 & 24.3 & 0.07 & $1.18 \pm 0.28$ \\
\hline Agarose (1.5\%) & $22^{\circ} \mathrm{C}$ & 13.3 & 13.4 & 0.05 & $2.25 \pm 0.67^{d}$ \\
\hline PL161 & $25^{\circ} \mathrm{C}$ & 42.7 & 61.2 & 18.5 & $48.8 \pm 2.5$ \\
\hline PL161 (15\%) & $40^{\circ} \mathrm{C}$ & 64.2 & 70.2 & 6.1 & $26.0 \pm 1.1$ \\
\hline DPPC:Ch & $25^{\circ} \mathrm{C}$ & 46.6 & 51.4 & 4.8 & $3.68 \pm 1.1^{\mathrm{e}}$ \\
\hline DPPC:Ch & $40^{\circ} \mathrm{C}$ & 31.2 & 39.0 & 7.8 & $10.85 \pm 3.2^{e}$ \\
\hline
\end{tabular}

${ }^{\mathrm{a}} \mathrm{T}_{1 \mathrm{D}}$ as determined by fitting a single exponential decay to Jeener-Broekaert data. Errors are $95 \%$ confidence levels of fit.

${ }^{\mathrm{b}} \mathrm{B}_{1}$ field (RMS) at $10.8 \mu \mathrm{T}$.

${ }^{\mathrm{c}} \mathrm{B}_{1}$ field (RMS) at $10.2 \mu \mathrm{T}$.

$\mathrm{d}_{10 \%}$ agarose in $\mathrm{D}_{2} \mathrm{O}(\mathrm{w} / \mathrm{w})$.

e Measured at 11.7 T. Other specimens and sample data were measured at 2.0 T.

multiple quantum and Zeeman order magnetization (39-41) during $\tau_{d}$. As above, wide-line proton NMR spec-

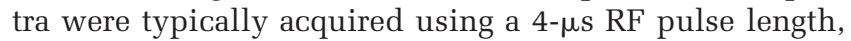
$\tau_{1}$ of $30 \mu \mathrm{s}, 100 \mathrm{kHz}$ spectral width, and $200 \mathrm{k}$ data points. Jeener-Broekaert data were reduced by summing the positive lobe of the dispersion-like spectrum. Data were obtained at $25^{\circ} \mathrm{C}$ and $40^{\circ} \mathrm{C}$ in samples of PL161, BSC WM, and BSC GM exchanged with $\mathrm{D}_{2} \mathrm{O}$. Other data were obtained from DPPC:Ch, 10\% agarose, cartilage, and $15 \%$ gelatin constructed in $\mathrm{D}_{2} \mathrm{O}$. Fitting of the decay of dipolar order, $\mathrm{T}_{1 \mathrm{D}}$, was performed with aid of the MATLAB Curve Fitting Tool using the trust-regionreflective algorithm. Errors of the fitting procedure are estimated as the $95 \%$ confidence limits. Decay of dipolar order was collected on a logarithmic time scale from $1 \mu \mathrm{s}$ to $1 \mathrm{~s}$ with 50 data points. The initial decay of magnetization is due either to fast dipolar decay, as in cartilage, or decay of the solid echo, as in Prolipid 161 and WM. For lipid samples, decay of magnetization between 0.6 $\mathrm{ms}$ and $1 \mathrm{~s}$ was used to calculate the predominately monoexponential $\mathrm{T}_{1 \mathrm{~d}}$ decay times.

\section{RESULTS}

MTR images at $20^{\circ} \mathrm{C}$ (Fig. 3a-c) and $40^{\circ} \mathrm{C}$ (Fig. 3d-f) for a specimen of BSC embedded in agarose gel are shown in Figure 3. At $20^{\circ} \mathrm{C}$, there is little difference between MTR and eMTR in either GM or WM, resulting in an ihMTR of $2.1 \pm 0.4$ for GM and $2.9 \pm 0.5$ for WM (Table 1). The most dramatic change in increasing the temperature to $40^{\circ} \mathrm{C}$ is a drop of MTR in WM from $65.3 \pm 2.2$ $\left(20^{\circ} \mathrm{C}\right)$ to $52.4 \pm 2.6\left(40^{\circ} \mathrm{C}\right)$. This drop in WM MTR causes ihMTR in WM to increase to $10.5 \pm 0.7$ at $40^{\circ} \mathrm{C}$. GM MTR also decreases $\left(63.7 \pm 0.8\right.$ at $20^{\circ} \mathrm{C}$ versus $59.7 \pm 0.9$ at $40^{\circ} \mathrm{C}$ ), leading to an ihMTR for $\mathrm{GM}$ at $40^{\circ} \mathrm{C}$ of $4.7 \% \pm 0.9 \%$. Agarose MT saturation is approximately $30 \%$ (Fig. 3a-e), regardless of temperature or RF saturation scheme.

Figure 4 presents the MT ( $\left.=\mathrm{M}_{\mathrm{sat}} / \mathrm{M}_{0}\right)$, eMT, and ihMT (=MT - eMT) frequency profiles (or z-spectra) for $15 \%$ w/w gelatin (Fig. 4a), 1.5\% agarose (Fig. 4b), and PL161 (Fig. 4c). The sharp feature between $\pm 100 \mathrm{~Hz}$ is direct saturation of water, and the broad (10-30 kHz) features are MT and eMT spectra. In gelatin and agarose (Fig. 4a,b), MT and eMT are identical and no ihMT is observed (Fig. 4, Table 1). Additional studies in crosslinked bovine serum albumin (data not shown) likewise reveal zero ihMT. PL161 shows increased eMT RF saturation beyond $\pm 3 \mathrm{kHz}$ (Fig. 4c). The difference between MT and eMT is the ihMT spectrum (Fig. 4c). The ihMT effect creates up to $25 \%$ enhancement at $\pm 12 \mathrm{kHz}$ relative to regular MT in this sample and under applied experimental conditions. The ihMT spectrum forms a broad doublet slightly asymmetric toward higher frequencies.

The temperature dependence of MT, eMT, and ihMT spectra for PL161 are shown in Figure 5. Raising the temperature of Prolipid increases the amount of regular MT (Fig. 5a) and significantly broadens the MT profile. The amount of eMT (Fig. 5b) also increases with increasing temperature, but the width of the eMT profile does not appreciably change. Comparison of Figs. 5a and 5b reveals that the spectral characteristics of the MT line shape change more with temperature than those of the eMT line shape. Much of the ihMT temperature dependence arises from changes in MT line shape. At $65^{\circ} \mathrm{C}$ (data not shown) MT becomes nearly identical to eMT. The amount of ihMT observed for PL161 (Fig. 5c) goes through a maximum near $25^{\circ} \mathrm{C}$ and gradually decreases with increasing temperature, nearly vanishing at $85^{\circ} \mathrm{C}$, when the MT z-spectrum collapses as the lipid melts. The off-resonance frequency at which the maximum amount of ihMT is observed increases with increasing temperature, from $10 \mathrm{kHz}$ at $5^{\circ} \mathrm{C}$ to $25 \mathrm{kHz}$ at $55^{\circ} \mathrm{C}$ (Fig. 5c). This trend is consistent with the increasing width of the regular MT spectrum with increasing temperature. In this PL161 sample, spin diffusion within the semisolid proton reservoir becomes more efficient with increasing temperature, MT and eMT become similar, and ihMT tends toward zero at higher temperatures.

Solid-state NMR spectra of PL161 in $\mathrm{D}_{2} \mathrm{O}$ (obtained with the sequence shown in Fig. 2c) are shown in Figure 6. Spectra of the broad semisolid components are shown 
FIG. 3. Percent MTR (a, d), $\operatorname{eMTR}(\mathbf{b}, \mathbf{e})$, and inMTR (c, f) of formalin-fixed $\mathrm{BSC}$ at $20^{\circ} \mathrm{C}(\mathrm{a}-\mathrm{C})$ and $40^{\circ} \mathrm{C}(\mathrm{d}-\mathrm{f})$. The amount of inMTR in WM (white arrow) changes significantly upon heating to $40^{\circ} \mathrm{C}$ compared with $\mathrm{GM}$ (black arrow). Grayscale images are \% MTR and color images are $\%$ inMTR.
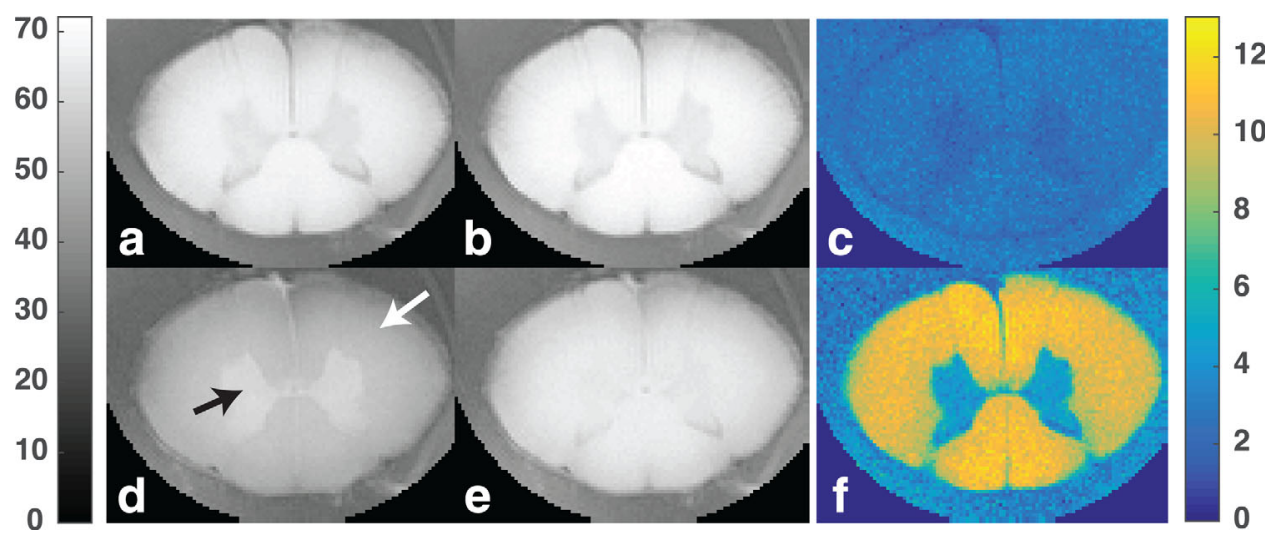

in Fig. 6a and narrow water (left) and methyl (right) components in 6b. Figure 6a illustrates inhomogeneous RF saturation of the semisolid PL161 proton spectrum. $\mathrm{RF}$ applied at $+10 \mathrm{kHz}$ (orange) preferentially saturates spins at positive offset, whereas RF applied at $-10 \mathrm{kHz}$ (blue) preferentially saturates spins at negative offset. Application at both positive and negative frequencies (dashed line) increases overall saturation of the PL161 protons. These same results are reflected in the MT spectra in Figure 6b. Residual water proton magnetization is equally reduced by either positive or negative RF (MT), but additional, enhanced MT occurs when dual-sided saturation is used (Fig. 6b). Methyl protons have minimal MT, because fast rotation about the three-fold symmetry axis decouples the methyl protons from the methylene protons to first order.

Figure 7 shows synthetic dipolar spectra generated by the difference between positive and negative RF saturation on the solid line for PL161 (Fig. 7a,c) and LLC (Fig. $7 \mathrm{~b}, \mathrm{~d}$ ) in $\mathrm{D}_{2} \mathrm{O}$ as a function of RF frequency from $\pm 100 \mathrm{~Hz}$ to $\pm 100 \mathrm{kHz}$. The images in Figures 7a,b and spectra in Figures 7c,d show that PL161, with 15-18 carbon atoms per aliphatic chain, has a much broader spectral width and much different saturation profile than the LLC sample, composed of molecules with 10 or 12 carbon atoms. The PL 161 spectra present characteristic features of overlapping Pake patterns with peak intensities at \pm 8 $\mathrm{kHz}$ and $\pm 17 \mathrm{kHz}$, respectively, whereas the LLC profile peaks at approximately $8 \mathrm{kHz}$. At large off-resonance fre- quencies $(>40 \mathrm{kHz})$, there is no MT and no ihMT. At small saturation frequencies $(<1 \mathrm{kHz}), \mathrm{RF}$ saturation is homogeneous. The amplitude of the saturation RF was $31 \mu \mathrm{T}$ for the PL161 sample and $1.32 \mu \mathrm{T}$ for the LLC sample. Although they occur at different RF amplitudes in each sample, these data are the maximum amount of ihMT obtained as a function of $B_{1}$ field strength (data not shown).

Subtraction of spectra obtained with $(+/+) 10 \mathrm{kHz}$ RF saturation from data obtained with $(-/-) 10 \mathrm{kHz}$ is shown for two temperatures for PL161 (Fig. 8a) and DPPC:Ch (Fig. 8b). The magnitude of the difference decreases with increasing temperature for PL161 but increases for DPPC:Ch. Figure 8a shows that raising the temperature from $25^{\circ} \mathrm{C}$ to $45^{\circ} \mathrm{C}$ reduces the asymmetric $\mathrm{RF}$ saturation in PL161, consistent with the reduction in ihMT seen in Figure 5 between these two temperatures. In contrast to PL161, Figure $8 \mathrm{~b}$ shows that increasing temperature increases asymmetric RF saturation in DPPC:Ch, consistent with the changes observed for BSC (Fig. 3). Furthermore, the dipole pattern is wide, with peak frequency at $\pm 17 \mathrm{kHz}$. Interestingly, the width of the PL161 saturation spectrum does not appreciably change with increasing temperature, whereas the integrated intensity decreases.

Proton $\mathrm{T}_{1 \mathrm{D}}$ was measured for BSC WM in $\mathrm{D}_{2} \mathrm{O}$ and for PL161 at 25 and $40^{\circ} \mathrm{C}$ and for hyaline cartilage at $25^{\circ} \mathrm{C}$. Data were fitted to a monoexponential decay. The results of the fit for Jeener-Broekaert measurements (Fig. 9)
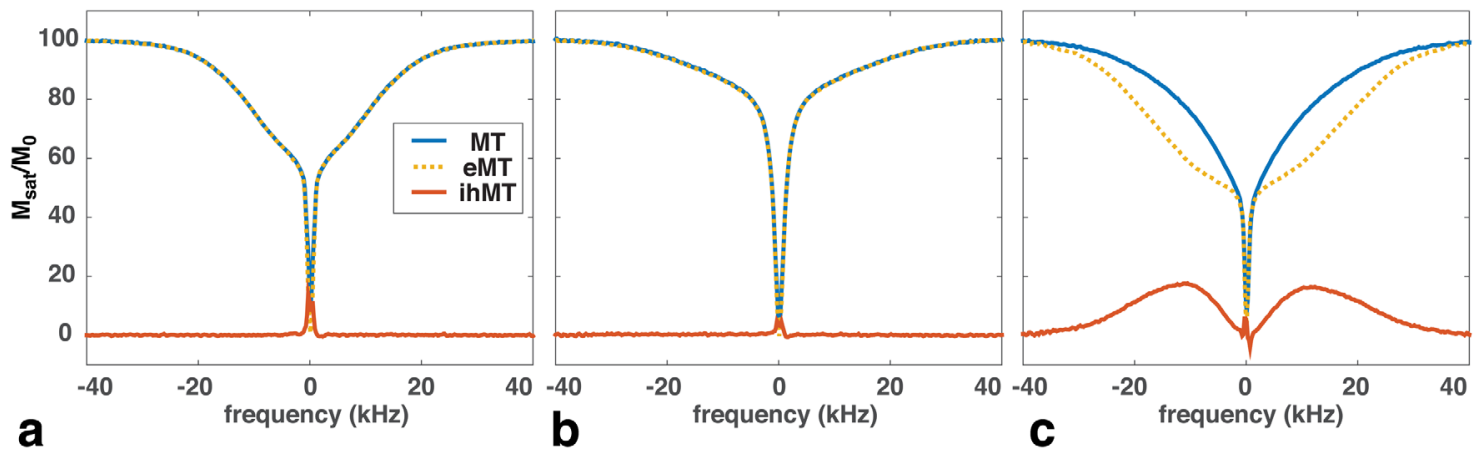

FIG. 4. MT (solid blue), eMT (dashed yellow), and inMT (solid rust) in gelatin (a), agarose (b), and prolipid 161 (c). ihMT is effectively zero for gelatin and agarose and approximately $20 \%$ at $10 \mathrm{kHz}$ in PL161. Lamellar lipid bilayers, such as in PL161 or in WM, create structural and dynamic conditions favorable for generation of inMT. 

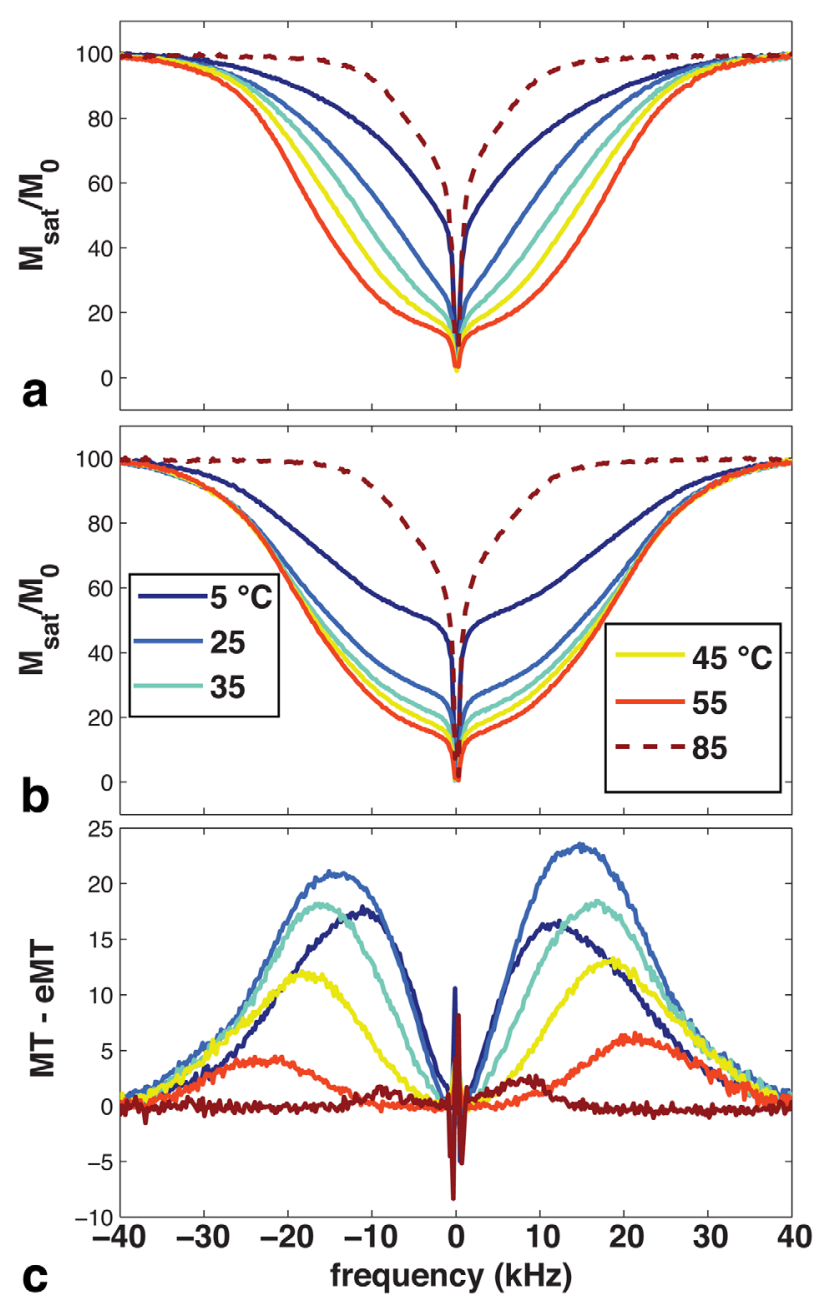

FIG. 5. Illustration of amplitude and width of MT (a), eMT (b), and inMT (c) profiles in 15\% PL161 as function of temperature. Data were acquired from $5^{\circ} \mathrm{C}$ to $85^{\circ} \mathrm{C}$ as indicated in panel $\mathrm{b}$.

clearly show that dipolar order decayed very quickly in the specimen of hyaline cartilage, where protons in the protein and polysaccharide matrix are strongly coupled and homogeneously broadened. In addition, dipolar order decayed more slowly at $40^{\circ} \mathrm{C}$ than at $25^{\circ} \mathrm{C}$ for BSC WM, whereas the trend was reversed for PL161, with much slower decay at $25^{\circ} \mathrm{C}$ versus $40^{\circ} \mathrm{C}$. These trends are consistent with temperature studies of saturation spectra summarized in Figure 8. As outlined by Jeener and Broekaert (41) and discussed by Yang and Schleich (39), it is necessary to wait several hundred microseconds to avoid contaminating dipolar decay with decay of the proton solid echo. Even when $\mathrm{T}_{1 \mathrm{D}}$ times in lipids are of order $1 \mathrm{~ms}$, as in $\mathrm{WM}$ at $25^{\circ} \mathrm{C}$, sufficient magnetization remains at $600 \mu \mathrm{s}$ to obtain a reasonably accurate estimate of $\mathrm{T}_{1 \mathrm{D}}$.

MTR, eMTR, and ihMTR values at $(+/+) 10$ and (+/-) $10 \mathrm{kHz}$ of all studies are summarized in Table 1 . In addition, proton $\mathrm{T}_{1 \mathrm{D}}$ estimates for $\mathrm{WM}, \mathrm{GM}$, gelatin, agarose, PL161, and DPPC:Ch are included. ihMT enhancement is largest in lipid-rich systems such as WM, $25^{\circ} \mathrm{C}$ PL161, and $40^{\circ} \mathrm{C}$ DPPC:Ch. Proton $\mathrm{T}_{1 \mathrm{D}}$ values follow the same content-specific trend, and in general ihMTR is largest (above $10 \%$ ) when proton $\mathrm{T}_{1 \mathrm{D}}$ is longest (of order 10 ms). ihMTR values presented in Table 1 are linearly correlated with measured values of proton $\mathrm{T}_{1 \mathrm{D}}$ (Pearson $\rho=0.934, P=7.6410^{-5}$ ).

\section{DISCUSSION}

These results demonstrate that ihMT is preferentially enhanced in WM, sensitive to temperature changes, observed primarily in lipid systems, a function of lipid chain length and molecular rigidity, and intimately related to proton $\mathrm{T}_{1 \mathrm{D}}$. ihMTR for all samples and temperatures are positively correlated with proton $\mathrm{T}_{1 \mathrm{D}}$. We find that an ihMTR image is effectively a proton $\mathrm{T}_{1 \mathrm{D}}$ weighted image, where long proton $\mathrm{T}_{1 \mathrm{D}}$ times lead to increased ihMTR. $\mathrm{T}_{1 \mathrm{D}}$ is a function of both dipolar coupling strength and molecular motion occurring at the local field, $\omega_{\text {loc }}$, and is sensitive to slow motions of order $\mathrm{kHz}$ (42-44). These properties make ihMT contrast sensitive to the slow molecular dynamics within the myelin membrane of WM.

As seen above, ihMT is generated when proton $\mathrm{T}_{1 \mathrm{D}}$ times are longer than several milliseconds. For a molecular system to have long proton $\mathrm{T}_{1 \mathrm{D}}$ times, three features must be present. First, there must be an intramolecular dipolar coupling, i.e., the inhomogeneous part of the dipolar Hamiltonian, $I_{1}^{z} I_{2}^{z}\left(1-3 \cos ^{2} \theta(t)\right)$, cannot be averaged to zero. Second, intermolecular spin-spin

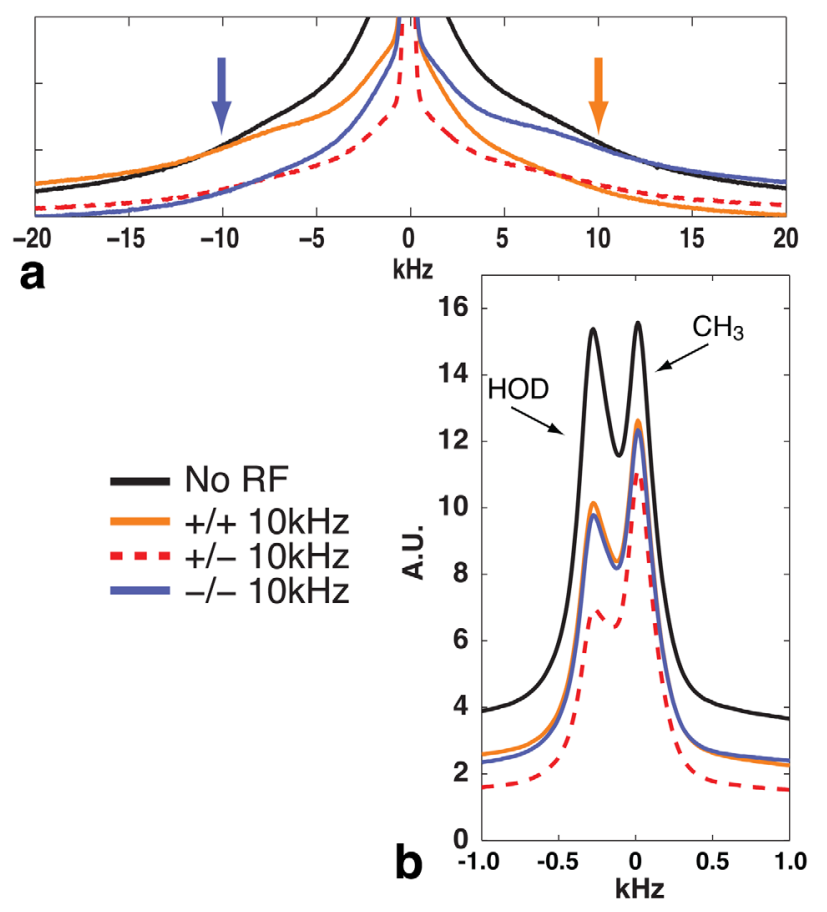

FIG. 6. Solid-state ${ }^{1} \mathrm{H}$ NMR spectra of PL161 in $\mathrm{D}_{2} \mathrm{O}$ with singlesided and double-sided RF saturation. Wideline NMR spectra (a) show asymmetric saturation of the PL161 semisolid line if RF is applied at $+10 \mathrm{kHz}$ (orange line and arrow $[+/+]$ ) or at $-10 \mathrm{kHz}$ (purple line and arrow [-/-]). (b) Residual water protons (HOD) have significant MT whereas methyl protons (upfield, or right of HOD) do not participate in MT. Saturation at both $+10 \mathrm{kHz}$ and $-10 \mathrm{kHz}$ RF (+/-) creates additional suppression of the semisolid line (dashed red line in panel a) and enhanced MT to HOD (dashed red line in panel b). 
FIG. 7. Asymmetric, offsetdependent RF saturation in PL161: $\mathrm{D}_{2} \mathrm{O}(\mathbf{a}, \mathbf{c})$ and LLC (b, d). These data are the difference between spectra with positive RF saturation $(+/+)$ and negative RF saturation (-/-) as a function of RF frequency. Individual difference spectra are extracted in (c,d) at five frequencies (also indicated by colored lines in panels $a$ and $b$ ).

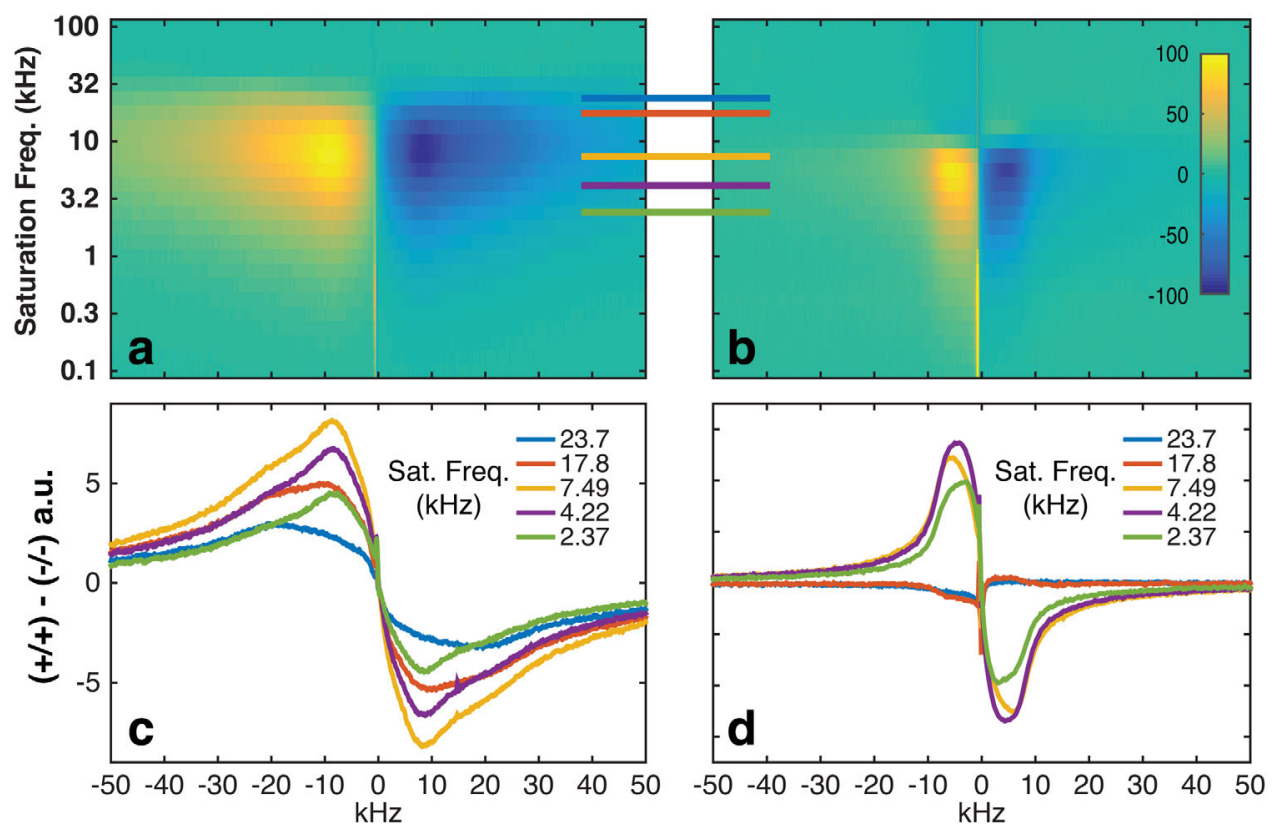

interactions need to be decoupled by motions so that isolated systems of dipolar-coupled spins are present. If this does not occur, spin diffusion will quickly mix the two degenerate Zeeman states, as in cartilage, via the "flip-flop" term of the homonuclear dipolar Hamiltonian, $-\left\{I_{1}^{+} I_{2}^{-}-I_{1}^{-} I_{2}^{+}\right\} \quad\left(1-3 \cos ^{2} \theta(t)\right)$, and cause significant homogeneous line broadening. Homogenous broadening will destroy dipolar order and suppress creation of ihMT. Finally, to generate MT, a magnetic, atomic, or molecular exchange between the semisolid component in tissue and water must exist.

\section{ihMT Imaging in BSC Tissue}

BSC imaging results show that ihMTR at physiologic temperature is greater and proton $\mathrm{T}_{1 \mathrm{D}}$ is longer in WM than in GM. These results are likely due to both the multilamellar structure and the relatively low protein content in myelin (30\%) with respect to GM (55\%) $(16,17,45)$. MT in lipids occurs by proton exchange between water and exchangeable hydroxyl groups in lipids $(18,21)$. The higher protein content of GM provides additional (regular) MT between water and homogenously broadened protein components. In addition, integral membrane proteins increase lipid rigidity in annular regions near the protein (46) and create additional homogeneous broadening (47). The combination of these two aspects of protein in membranes will decrease proton $\mathrm{T}_{1 \mathrm{D}}$ and lessen the amount of ihMT observed in GM relative to WM. The relatively high absolute MTR in GM observed for the fixed BSC sample could be related to extensive cross-linking of proteins upon formalin fixation that increases the amount of homogeneously broadened semisolid component $(18,48)$.

MT, eMT, and ihMT images of BSC at $20^{\circ} \mathrm{C}$ and $40^{\circ} \mathrm{C}$ further support the hypothesis that changes in membrane dynamics are responsible for differences in ihMT. At $20^{\circ} \mathrm{C}$, MTR and eMTR for WM and GM are similar, and ihMTR is nearly zero. WM at $40^{\circ} \mathrm{C}$, however, behaves differently. MTR decreases by $12.9 \%$ at $40^{\circ} \mathrm{C}$, whereas eMTR only decreases by $5.3 \%$. These changes show that the main sensitivity of ihMTR rests not in eMT, but in the MT signal. However, the eMT images are a critical component of the experiment, providing an image with effects of dipolar order removed and allowing computation of ihMT.

\section{Molecular Structure, Molecular Dynamics, and ihMT}

Our results show that ihMT occurs in samples of PL161 in LLC and DPPC:Ch but not in agarose or gelatin. Temperature increases in PL161 change both the intensity and frequency spread of ihMT. These findings are consistent with inhomogeneous broadening of semisolid spectra with characteristic dipole order features. These features are known sensitive functions of membrane order and fluidity determined both by membrane constitution and their dynamic regimen $(14,15,49)$.

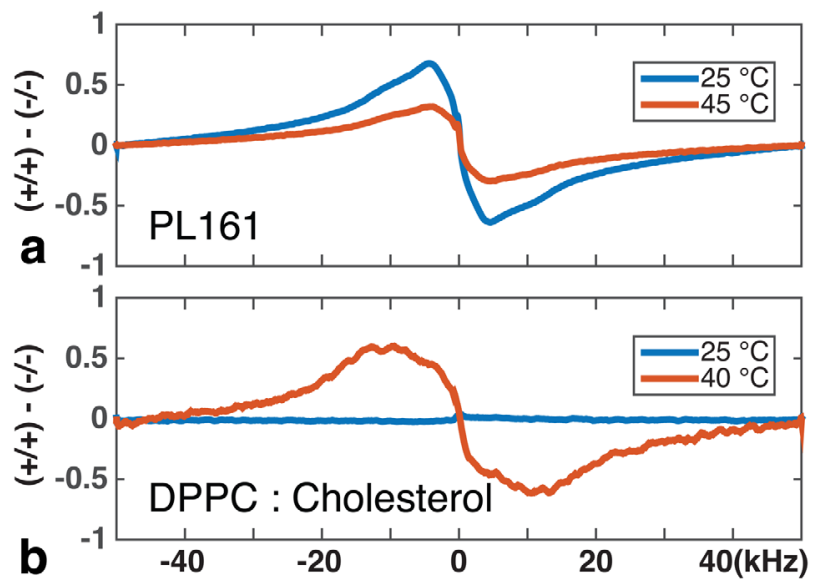

FIG. 8. Difference between positive $(+/+)$ and negative (-/-) RF saturation in PL161 (a) and DPPC:Ch (b) at $25^{\circ} \mathrm{C}$ versus $45^{\circ} \mathrm{C}$ for PL161 and $25^{\circ} \mathrm{C}$ versus $40^{\circ} \mathrm{C}$ for DPPC:Cho. 

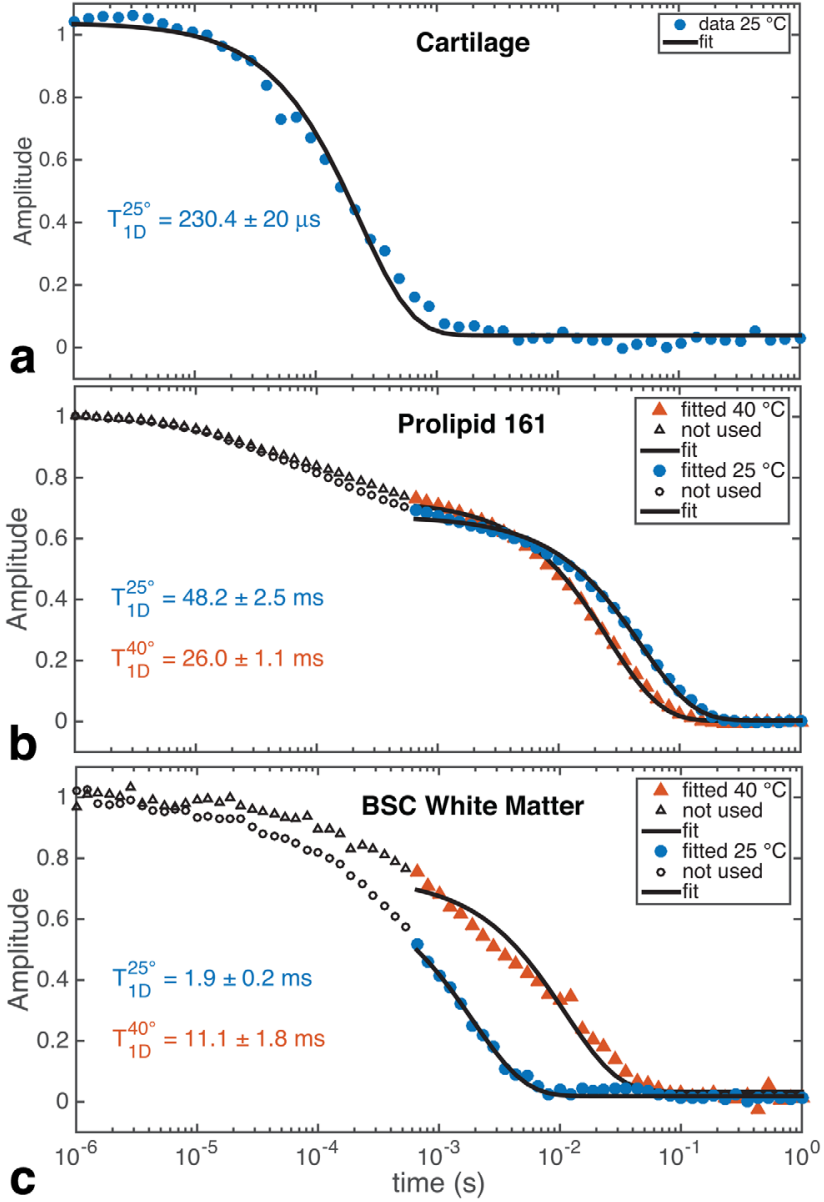

FIG. 9. Jeener-Broekaert decay of dipolar order in cartilage (a), PL161 (b), and WM (c) at $25^{\circ} \mathrm{C}$ (blue) and $40^{\circ} \mathrm{C}$ (red). A monoexponential relaxation function is fitted to the data to estimate $T_{1 \mathrm{D}}$ at $25^{\circ} \mathrm{C}\left(T_{1 D}^{25^{\circ}}\right.$, blue text) and $40^{\circ} \mathrm{C}\left(T_{1 D}^{40^{\circ}}\right.$, red text). These trends follow imaging and spectroscopy results seen in Figure 3c,f and Figure $5 \mathrm{c}$, respectively.

\section{Lipid Systems}

The structure and dynamics of lipid and liquid crystal systems fulfill the criteria for inhomogeneous broadening described above. Fast motions in lipids about the lipid carbon chain partially average the intramolecular dipolar interaction, and molecular diffusion eliminates intermolecular dipolar interactions (15,50). These motions give rise to the super-Lorentzian line shape in lipids, which is a projection of the $\mathrm{CH}_{2}$ dipolar interactions onto the lipid director axis, creating broad semisolid resonance (51).

Consistent with the previous dynamic NMR results $(13,52)$, DPPC:Ch is the most rigid of the lipids systems in this study. The structure of DPPC:Ch limits molecular rotations and translations relative to LLC and PL161, creating an environment for more efficient spin diffusion and reduced overall ihMT. PL161 is composed of amphiphilic molecules and alcohols with long, single carbon chains with exchangeable hydroxyl groups, whereas DPPC:Ch has two palmitoyl carbon chains at the glycerol linkage and a relatively rigid, planar cholesterol with exchangeable hydroxyl protons. This interpretation is consistent with the relatively large ihMT observed in PL161 (up to 20\% and greater than any other system studied here) and makes PL161 a good phantom material to test ihMT pulse sequences. However, DPPC:Ch and other phospholipid and sterol variations more accurately mimic biological membranes. Future quantitative studies of ihMT and $\mathrm{T}_{1 \mathrm{D}}$ as a function of membrane constituents and temperature will determine which molecular and dynamic components generate and suppress ihMT in vivo.

The relationship between ihMT peak intensity and solid-state NMR line shapes is further elucidated by the difference between measured dipole spectra with positive $(+/+)$ and negative $(-/-)$ RF saturation shown at two samples, PL161, and LLC. Higher rigidity of PL161 lipids and DPPC:Ch membranes compared with LLC results in the broader width of their saturation profiles when compared with LLC.

The ability to generate synthetic dipolar spectra is related to the length of proton $\mathrm{T}_{1 \mathrm{D}}$. If $\mathrm{T}_{1 \mathrm{D}}$ is long, the single-sided RF saturation selectively saturates one side of the spectrum. If $\mathrm{T}_{1 \mathrm{D}}$ is short, spin diffusion will homogeneously couple one side to the other. The amplitude of these spectra decrease with increasing temperature for PL161 and increase for DPPC:Ch. These changes correlate with measured $\mathrm{T}_{1 \mathrm{D}}$ times and ihMT values. This behavior is consistent with the molecular dynamics of PL161 and DPPC:Ch existing on different sides of a $\mathrm{T}_{1 \mathrm{D}}$ maximum as temperature is increased. The observed dynamic changes are likely reflecting alterations in membrane structure and fluidity expected during phase transition between disordered and ordered lipid phases (49,52). Temperature effects on DPPC:Ch saturation spectra and $\mathrm{T}_{1 \mathrm{D}}$ times are also consistent with changes observed in BSC. In PL161, the dynamic trends are reversed, with lower ihMT and shorter proton $\mathrm{T}_{1 \mathrm{D}}$ times at higher temperatures. The preserved width of saturation spectra observed with increasing temperature is a likely indicator that measured dynamic $\mathrm{T}_{1 \mathrm{D}}$ changes are related to changes in the slow rates of molecular motion within lipid membrane.

The intensity of the ihMT signal and the frequency of the maximum ihMT also change with temperature for PL161. These findings can be explained if we assume that at room temperature there are several components that contribute to ihMT, some with small and some with large order parameters, as evident from the corresponding saturation spectra. The components with larger order parameters will be more rigid than the components with the smaller order parameters. As the temperature increases, components with the small order parameter become more fluid-like and are removed from the ihMT spectrum. The components that remain are more rigid. Distance of carbon from the water bilayer interface determines the magnitude of the order parameter $(13,52)$. Those carbons at the surface are more rigid and have a higher order parameter than those buried within the lipid bilayer.

\section{Proteins and Polysaccharides}

The semisolid proton resonance of immobilized proteins is homogeneously broadened by efficient spin diffusion $(8,9)$, and minimal ihMT is expected. Proton $\mathrm{T}_{1 \mathrm{D}}$ is $1.18 \pm 0.28 \mathrm{~ms}$ in gelatin, $2.25 \pm 0.67 \mathrm{~ms}$ for agarose, and $230.4 \pm 20 \mu \mathrm{s}$ in cartilage, similar to values reported 
previously in proteins $(53,54)$ or polysaccharides (39). Lack of ihMT generation for both gelatin and agarose could possibly be explained by the relatively long, 5-ms $\mathrm{RF}$ pulse used in the ssMT experiment to generate MT and eMT. To test for ihMT under different experimental conditions, a cosine modulated RF pulse train with instantaneous, double-sided RF saturation was used. These experiments also failed to generate ihMT in protein or agarose samples (data not shown). These results support the hypothesis that ihMT occurs only in spin systems with inhomogeneous broadening and long proton $\mathrm{T}_{1 \mathrm{D}}$ times.

In addition to lipids, other molecular systems have very long proton $\mathrm{T}_{1 \mathrm{D}}$ times (39) and will be amenable to coupling and decoupling of Zeeman and dipolar order by single-sided and double-sided RF saturation, respectively. It may be that there are molecular constructions in vivo other than $\mathrm{WM}$ that generate long $\mathrm{T}_{1 \mathrm{D}}$ times and large ihMT signals. In principle, ihMT could be present in protein or polysaccharide systems if certain structural and dynamic conditions existed to create sufficiently long proton $\mathrm{T}_{1 \mathrm{D}}$ times, though we find no evidence of that here. Additional studies are needed to establish a quantitative relation between ihMT and specific tissue components. The intensity of ihMTR images appears to be sensitive to myelin content in tissue. WM has the highest ihMTR signal, more than twice as large as that for GM. A valid question to ask is: "Is the intensity of an ihMTR image proportional to myelin?" That question remains open for investigation by quantitative ihMT studies and corresponding quantitative model fitting. The correlation between of ihMTR and myelin content remains to be established by careful imaging and histology studies.

\section{CONCLUSION}

This study demonstrates that ihMT contrast is defined by proton $\mathrm{T}_{1 \mathrm{D}}$ times in lipid and phospholipid systems, which are abundant in myelin membranes. A promising feature for future clinical application of ihMT imaging is that proton $\mathrm{T}_{1 \mathrm{D}}$ is sensitive to slow motions of order $\mathrm{kHz}$; a dynamic window not visualized by conventional $\mathrm{T}_{1}$ or $\mathrm{T}_{2}$ imaging. Alterations of slow membrane dynamics may precede changes in myelin content under certain disease processes.

\section{REFERENCES}

1. Henkelman RM, Huang XM, Xiang QS, Stanisz GJ, Swanson SD, Bronskill MJ. Quantitative Interpretation of Magnetization-Transfer. Magn Reson Med 1993;29:759-766.

2. Filippi M, Campi A, Dousset V, Baratti C, Martinelli V, Canal N, Scotti G, Comi G. A magnetization transfer imaging study of normal-appearing white matter in multiple sclerosis. Neurology 1995;45:478-482.

3. Wolff SD, Balaban RS. Magnetization transfer contrast (Mtc) and tissue water proton relaxation in vivo. Magn Reson Med 1989;10:135-144.

4. Sled JG, Pike GB. Quantitative imaging of magnetization transfer exchange and relaxation properties in vivo using MRI. Magn Reson Med 2001;46:923-931.

5. van Zijl PCM, Zhou J, Mori N, Payen JF, Wilson D, Mori S. Mechanism of magnetization transfer during on-resonance water saturation. A new approach to detect mobile proteins, peptides, and lipids. Magn Reson Med 2003;49:440-449.

6. Janve VA, Zu Z, Yao S-Y, Li K, Zhang FL, Wilson KJ, Ou X, Does MD, Subramaniam S, Gochberg DF. The radial diffusivity and mag- netization transfer pool size ratio are sensitive markers for demyelination in a rat model of type III multiple sclerosis (MS) lesions. Neuroimage 2013;74:298-305.

7. Turati L, Moscatelli M, Mastropietro A, et al. In vivo quantitative magnetization transfer imaging correlates with histology during deand remyelination in cuprizone-treated mice. NMR Biomed 2015;28: 327-337.

8. Akasaka K. Longitudinal relaxation of protons under cross saturation and spin diffusion. J Magn Reson 1981;45:337-343.

9. Kalk A, Berendsen HJC. Proton magnetic relaxation and spin diffusion in proteins. J Magn Reson 1976;24:343-366.

10. Wennerstrom H. Proton nuclear magnetic resonance lineshapes in lamellar liquid crystals. Chem Phys Lett 1973;18:41-44.

11. Brown MF. Deuterium relaxation and molecular dynamics in lipid bilayers. J Magn Reson 1979;35:203-215.

12. Brown MF, Ribeiro AA, Williams GD. New view of lipid bilayer dynamics from $\mathrm{H}-2$ and C-13 NMR relaxation-time measurements. Proc Natl Acad Sci U S A 1983;80:4325-4329.

13. Brown MF, Nevzorov AA. H-2-NMR in liquid crystals and membranes. Colloids Surf A Physicochem Eng Asp 1999;158:281-298.

14. Davis JH. Deuterium magnetic resonance study of the gel and liquid crystalline phases of dipalmitoyl phosphatidylcholine. Biophys J 1979;27:339-358.

15. Davis JH. The description of membrane lipid conformation, order and dynamics by H-2-NMR. Biochim Biophys Acta 1983;737:117-171.

16. Obrien JS, Sampson EL. Fatty acid and fatty aldehyde composition of major brain lipids in normal human gray matter white matter and myelin. J Lipid Res 1965;6:545-551.

17. Obrien JS, Sampson EL. Lipid composition of normal human braingray matter, white matter, and myelin. J Lipid Res 1965;6:537-545.

18. Koenig SH. Cholesterol of myelin is the determinant of gray-white contrast in MRI of brain. Magn Reson Med 1991;20:285-291.

19. Fralix TA, Ceckler TL, Wolff SD, Simon SA, Balaban RS. Lipid bilayer and water proton magnetization transfer: effect of cholesterol. Magn Reson Med 1991;18:214-223.

20. Ceckler TL, Wolff SD, Yip V, Simon SA, Balaban RS. Dynamic and chemical factors affecting water proton relaxation by macromolecules. J Magn Reson 1992;98:637-645.

21. Kucharczyk W, Macdonald PM, Stanisz GJ, Henkelman RM. Relaxivity and magnetization transfer of white matter lipids at MR imaging: importance of cerebrosides and pH. Radiology 1994;192:521-529.

22. Forbes J, Husted C, Oldfield E. High-field, high-resolution proton magic-angle sample-spinning nuclear magnetic-resonance spectroscopic studies of gel and liquid-crystalline lipid bilayers and the effects of cholesterol. J Am Chem Soc 1988;110:1059-1065.

23. Polozov IV, Gawrisch K. Characterization of the liquid-ordered state by proton MAS NMR. Biophys J 2006;90:2051-2061.

24. Pake GE. Nuclear resonance absorption in hydrated crystals: fine structure of the proton line. J Chem Phys 1948;16:327-336.

25. Varma G, Duhamel G, de Bazelaire C, Alsop DC. Magnetization transfer from inhomogeneously broadened lines: a potential marker for myelin. Magn Reson Med 2015;73:614-622.

26. Girard OM, Prevost VH, Varma G, Cozzone PJ, Alsop DC, Duhamel G. Magnetization transfer from inhomogeneously broadened lines (ihMT): experimental optimization of saturation parameters for human brain imaging at 1.5 Tesla. Magn Reson Med 2015;73:2111-2121.

27. Yeung HN, Adler RS, Swanson SD. Transient decay of longitudinal magnetization in heterogeneous spin systems under selective saturation. 4. Reformulation of the spin bath model equations by the Redfield-Provotorov theory. J Magn Reson Series A 1994;106:37-45.

28. Adler RS, Swanson SD, Yeung HN. A three-component model for magnetization transfer. Solution by projection-operator technique, and application to cartilage. J Magn Reson Series B 1996;110:1-8.

29. Lee J-S, Khitrin AK, Regatte RR, Jerschow A. Uniform saturation of a strongly coupled spin system by two-frequency irradiation. J Chem Phys 2011;134:234504.

30. Lee J-S, Regatte RR, Jerschow A. Isolating chemical exchange saturation transfer contrast from magnetization transfer asymmetry under two-frequency rf irradiation. J Magn Reson 2012;215:56-63.

31. Provotorov BN. Magnetic resonance saturation in crystals. Journal of Experimental and Theoretical Physics 1962;14:1126-1131.

32. Goldman M. Spin temperature and nuclear magnetic resonance in solids. Oxford, UK: Clarendon Press; 1970. 246 p.

33. Morrison C, Stanisz G, Henkelman RM. Modeling magnetization transfer for biological-like systems using a semisolid pool with a 
super-Lorentzian lineshape and dipolar reservoir. J Magn Reson Series B 1995;108:103-113.

34. Varma G, Girard OM, Prevost VH, Grant AK, Duhamel G, Alsop DC. Interpretation of magnetization transfer from inhomogeneously broadened lines (ihMT) in tissues as a dipolar order effect within motion restricted molecules. J Magn Reson 2015;260:67-76.

35. Clough S. Nuclear spin relaxation in a rotating tilted reference frame. Phys Rev 1967;153:355-357.

36. Mackay AL, Burnell EE, Nichol CP, Weeks G, Bloom M, Valic MI. Effect of viscosity on width of methylene proton magnetic-resonance line in sonicated phospholipid bilayer vesicles. FEBS Lett 1978;88: 97-100.

37. Alderman DW, Grant DM. Efficient decoupler coil design which reduces heating in conductive samples in superconducting spectrometers. J Magn Reson 1979;36:447-451.

38. Swanson SD. Broad-band excitation and detection of cross-relaxation NMR spectra. J Magn Reson 1991;95:615-618.

39. Yang H, Schleich T. Modified Jeener solid-echo pulse sequences for the measurement of the proton dipolar spin-lattice relaxation-time ( $\mathrm{T}$ 1d) of tissue solid-like macromolecular components. J Magn Reson Series B 1994;105:205-210.

40. Vold RL, Dickerson WH, Vold RR. Application of the JeenerBroekaert pulse sequence to molecular dynamics studies in liquid crystals. J Magn Reson 1981;43:213-223.

41. Jeener J, Broekaert P. Nuclear magnetic resonance in solids: thermodynamic effects of a pair of RF pulses. Phys Rev 1967;157:232-240.

42. Resing HA. NMR relaxation in adamantane and hexamethylenetetramine: diffusion and rotation. Molecular Crystals and Liquid Crystals 1969;9:101-132.

43. Ueda T, Takeda S, Nakamura N, Chihara H. Molecular motion and phase changes in long-chain solid normal alkanes as studied by H-1 and C-13 NMR. Bulletin of the Chemical Society of Japan 1991;64: 1299-1304.
44. Zamar RC, Mensio O. Nuclear magnetic resonance proton dipolar order relaxation in thermotropic liquid crystals: a quantum theoretical approach. J Chem Phys 2004;121:11927-11941.

45. Siegel GJ, Albers RW, Brady ST, Price DL. Basic neurochemistry: molecular, cellular, and medical aspects. Burlington, London: Elsevier Academic; 2006. $992 \mathrm{p}$.

46. Lee AG. Lipid-protein interactions in biological membranes: a structural perspective. Biochim Biophys Acta 2003;1612:1-40.

47. Simons K, Ikonen E. Functional rafts in cell membranes. Nature 1997;387:569-572.

48. Koenig SH, Brown RD, Ugolini R. Magnetization transfer in crosslinked bovine serum albumin solutions at $200 \mathrm{MHz}$ : a model for tissue. Magn Reson Med 1993;29:311-316.

49. Redondo-Morata L, Giannotti MI, Sanz F. Influence of cholesterol on the phase transition of lipid bilayers: a temperature-controlled force spectroscopy study. Langmuir 2012;28:12851-12860.

50. Urbina JA, Moreno B, Arnold W, Taron CH, Orlean P, Oldfield E. A carbon-13 nuclear magnetic resonance spectroscopic study of interproton pair order parameters: a new approach to study order and dynamics in phospholipid membrane systems. Biophys J 1998;75: 1372-1383.

51. Davis JH, Auger M, Hodges RS. High resolution H-1 nuclear magnetic resonance of a transmembrane peptide. Biophys J 1995;69:1917-1932.

52. Trouard TP, Nevzorov AA, Alam TM, Job C, Zajicek J, Brown MF. Influence of cholesterol on dynamics of dimyristoylphosphatidylcholine bilayers as studied by deuterium NMR relaxation. J Chem Phys 1999;110:8802-8818.

53. Gaspar R, Andrew ER, Bryant DJ, Cashell EM. Dipolar relaxation and slow molecular motions in solid proteins. Chem Phys Lett 1982;86: 327-330.

54. Danek AN, Bryant RG. Decay of dipolar order in diamagnetic and paramagnetic proteins and protein gels. J Magn Reson 2000;143: 35-38. 